\title{
Nein zur Verstaatlichung und Bürokratie im Gesundheitswesen
}

Schweizerische Zahnärzte-Gesellschaft SSO

Korrespondenz:

Presse- und Informationsdienst der Schweizerischen ZahnärzteGesellschaft SSO

Postgasse 19

Postfach

CH-3000 Bern 8

Tel. 0313102080

Fax 0313102082

Internet: www.sso.ch
Die Schweizerische Zahnärzte-Gesellschaft SSO wird das von der Verbindung der Schweizer Ärzte FMH beschlossene Referendum gegen die 2. Teilrevision des Krankenversicherungsgesetzes unterstützen. Aus der Sicht der Zahnärzteschaft enthält die Revisionsvorlage starke Verstaatlichungstendenzen, führt zu einer noch weiter aufgeblähten Bürokratie und letztlich zu einem Absinken der Qualität medizinischer Leistungen - allen gegenteiligen Beteuerungen zum Trotz.

Was den Versicherten heute zugemutet werden soll, ist nicht mehr ehrlich. Wenn der Eindruck erweckt wird, man könne die Spitzenleistungen des schweizerischen Gesundheitswesens auch zu einem wesentlich günstigeren Preis haben, so ist dies schlicht nicht wahr. Medizin, Medizintechnik und Pharmazie versprechen für die nächsten Jahre und Jahrzehnte Fortschritte sowohl in Lebensqualität und Lebenserwartung, auf die - und dies zu Recht - kein Patient wird verzichten wollen. Weitere Kostensteigerungen im Gesundheitswesen sind deshalb unvermeidlich. In dieser Situation wären strikte Massnahmen angebracht: Das System der sozialen Krankenversicherung hat sich auf medizinisch nötige und wirksame Leistungen zu beschränken. Die Franchisen sind so zu erhöhen, dass die Patienten die ihnen wirtschaftlich zumutbaren Kosten selbst übernehmen. Im Gegenzug sind finanziell Schwache wirksam und administrativ einfach zu entlasten. Leistungserbringer, die das Versicherungssystem zu ihrem Vorteil ausnutzen, sind aus diesem System zu entfernen. Die Erfahrungen im Bereich der Zahnmedizin zeigen, dass so vom medizinischen Fortschritt profitiert werden kann, ohne dass eine unverantwortliche Kostensteigerung stattfindet.

Das System der sozialen Krankenversicherung ist in der Schweiz seit jeher staatlich gesteuert. Der in der Politik immer wieder propagierte «freie Wettbewerb», der alle Kostenprobleme lösen soll, ist eine Illusion. So lange Leistungserbringer wie Ärzte oder Spitäler ohne Vertrag mit den Krankenkassen keine Überlebenschance haben, kann von freiem Wettbewerb oder von Marktwirtschaft keine Rede sein. Die heute vorgesehenen Massnahmen - vor allem die Einschränkung der freien Arztwahl des Patienten - zementieren lediglich den Einfluss der Krankenkassen im Markt und führen zu einer Billigmedizin. 